\title{
Distributed Control of Wind Farm Power Set Points to Minimise Fatigue Loads
}

\author{
Adam Stock ${ }^{1}$, Matthew Cole ${ }^{2}$, William Leithead ${ }^{3}$, and Lindsey Amos ${ }^{4}$
}

\begin{abstract}
The quantity and size of wind farms continue to grow as countries around the world strive to meet ambitious targets for renewable electricity generation such as the UK governments Net Zero target of increasing offshore wind energy from current levels (circa $6 \mathrm{GW}$ ) to circa $75 \mathrm{GW}$ by 2050. With increasing size and quantity of wind farms, there is a growing requirement to use wind farm level control both to help with grid integration and to minimise the loads on the turbines in the farm. In this paper, a methodology of distributing power set points through a wind farm to minimise the loads on the turbines whilst meeting a delta power set point for the farm is presented. The methodology in this paper uses a hierarchical control structure, in which a network wind farm controller calculates the required change in wind farm power and then passes this value on to a distributed controller that defines the change in power required from each wind turbine. The network wind farm controller calculates a delta change in wind farm power that the wind farm holds in reserve. The distributed controller allocates the reductions in power output by first setting a baseline reduction that considers the steady state tower loads. The baseline is then adjusted to meet the required change in power, distributing the additional change proportional to the square of each turbines estimated wind speed. Performance is assessed using the StrathFarm simulation tool. The wind turbine models incorporated into StrathFarm are sufficiently detailed to provide the tower, blade and drivetrain loads and the wind field model is sufficiently detailed to represent turbulence, wind shear and tower shadow and wakes and their interaction. The performance of the proposed wind farm controller is assessed for a range of wind conditions for a $4 \times 4$ wind farm of $5 \mathrm{MW}$ wind turbines. Both the accuracy of the change in power output from the wind farm and the change in turbines DELs are discussed. Depending on the wind conditions, the approach is found to reduce the tower and blade loads by about $10 \%$ in comparison to each turbine being allocated the same change in power. There is good accuracy in the change in power at higher wind speeds. Below rated wind speed, wake effects reduce the accuracy of the change in power.
\end{abstract}

\section{INTRODUCTION}

The trend in wind energy over recent years has been for wind turbine and wind farms to increase in size, especially in the offshore case. The increase in size of turbines and farms is driven by the increasing goals for renewable energy

\footnotetext{
${ }^{1}$ Adam Stock is a Knowledge Exchange Fellow in the Wind Energy and Control Centre, University of Strathclyde, Glasgow, Scotland, G1 2TB, UK adam.stockestrath.ac.uk

${ }^{2}$ Matthew Cole is a Researcher in the Wind and Marine Centre for Doctoral Training, University of Strathclyde, Glasgow, Scotland, G1 1XW, UK matthew. coledstrath.ac.uk

${ }^{3}$ William Leithead is a Professor in the Wind Energy and Control Centre, University of Strathclyde, Glasgow, Scotland, G1 2TB, UK w. leitheadestrath.ac.ukestrath.ac.uk

${ }^{4}$ Lindsey Amos is a Researcher in the Wind and Marine Centre for Doctoral Training, University of Strathclyde, Glasgow, Scotland, G1 1XW, UK lindsey.amosestrath.ac.uk
}

connection, of which wind is expected to play a large role given its relative maturity compared to other renewable technologies. As an example, the United Kingdom has laid out its "Net-Zero" plan for 2050, which aims to increase offshore wind energy from around 7GW today to around $75 \mathrm{GW}$ by 2050 . Such a large increase in the volume of wind energy connected to the grid (note that the peak demand for the UK in 2018 was $50.3 \mathrm{GW}$ [1]) will necessitate that wind farms no longer operate in a "greedy" fashion as is often currently the case, whereby all wind turbines aim to maximise their own energy capture up to their rated power without consideration of wider network or farm considerations. Instead, it is likely that wind farms will be required to contribute in a more meaningful way to grid balancing, and turbines will need to consider the impact of their strategy on structural loading. Contributing to the stability of the wider grid can be done by holding power in reserve, sometimes called "delta control". Fast and accurate delta control allows wind turbines and/or wind farms to contribute to ancillary services such as synthetic inertia or droop control through variation of the power held in reserve. Reducing the power output of wind turbines can be done in many ways, with different advantages and disadvantages in terms of the speed of response to changes in power and the loads induced upon the turbine [4]. In this paper, a methodology is presented that allows the wind farm to supply accurate delta control, whilst distributing the required change in power of each turbine between the turbines in an intelligent way to minimise the loads. In section II the methodology and tools required to investigate the problem are detailed. The wind farm layout and environmental conditions for the simulations are detailed in section III. In section IV the results of the simulations are discussed, with conclusions drawn in section V.

\section{Simulation Tools and Methodology}

In order to simulate the proposed wind farm control approach, a suitable wind farm simulation tool is required. Simulation of large numbers of wind turbines can be highly computationally demanding for high fidelity simulations such as those using blade element momentum theory (BEM) or CFD, however, running low fidelity simulations (such a simple power curve look up or a basic actuator disc model) does not provide a good enough assessment of the dynamics of the turbines and the loads on the turbines in order to accurately assess the loads resulting from the control action and site conditions simulated. In order to bridge the gap and allow multiple simulations to be run whilst still providing the necessary level of complexity to assess individual turbine 
dynamics and the relative loads induced through different control approaches, the medium fidelity "StrathFarm" tool has been developed. StrathFarm uses wind turbine models, developed over the past 20 years, which incorporate lumped parameter models of the drive-train and rotor, aerodynamic modelling that includes induction lag effects, and modelling of blade and tower loads validated against DNV GL's Bladed. The wind comprises a pre-processed correlated low frequency wind farm wind field, to which higher frequency effective wind field components are added locally to each turbine. Wakes can be modelled in two ways; an engineering model based on the work of Frandsen [5] or a higher fidelity method based on the WFSim model [2]. StrathFarm is uniquely placed in providing a simulation environment that allows medium fidelity simulations modelling turbine dynamics and wakes in turbulent winds to be run in better than real time for wind farms of up to 100 wind turbines. A schematic view of Strathfarm is presented in Fig. 1, with each of the major items discussed in more detail in the following subsections.

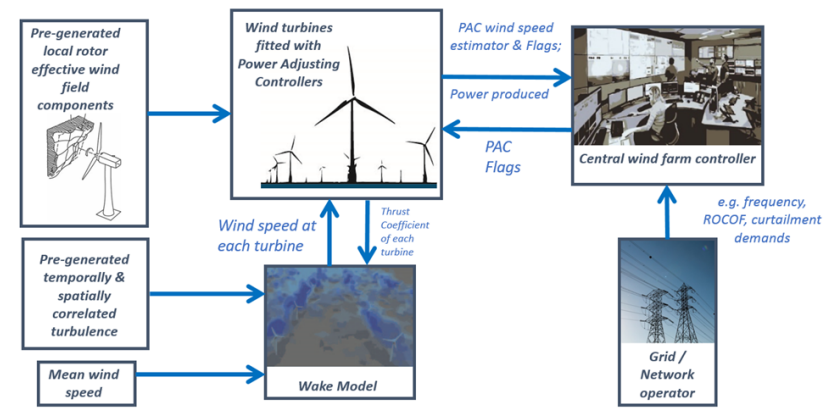

Fig. 1. StrathFarm schematic view

\section{A. Wind Field Model}

Wind turbines experience a time varying wind field consisting of variations that are both spatial and temporal. The wind speed has a mean value, about which speed varies via deterministic components, such as wind shear and stochastic components (turbulence). The stochastic elements of the wind speed variation can be considered as two separate components, one at low frequencies and the other at high frequencies. At low frequencies the wind speed is highly correlated across the whole rotor disc and so it is both possible and suitable to model the the low frequency component of the wind speed variation as a slowly varying scaler wind speed. In the case of StrathFarm, because the length scales of the low frequency components are similar to the distances separating turbines, each turbine in the wind farm (and often at some intermediary points between turbines in the wind farm) has a varying wind speed calculated via the Veers method [16] with a relatively low sampling time of the order of 1 second. In this manner the low frequency components of the wind are well modelled. For higher frequency components the correlation over distances similar to the separation of wind turbines in a wind farm is very
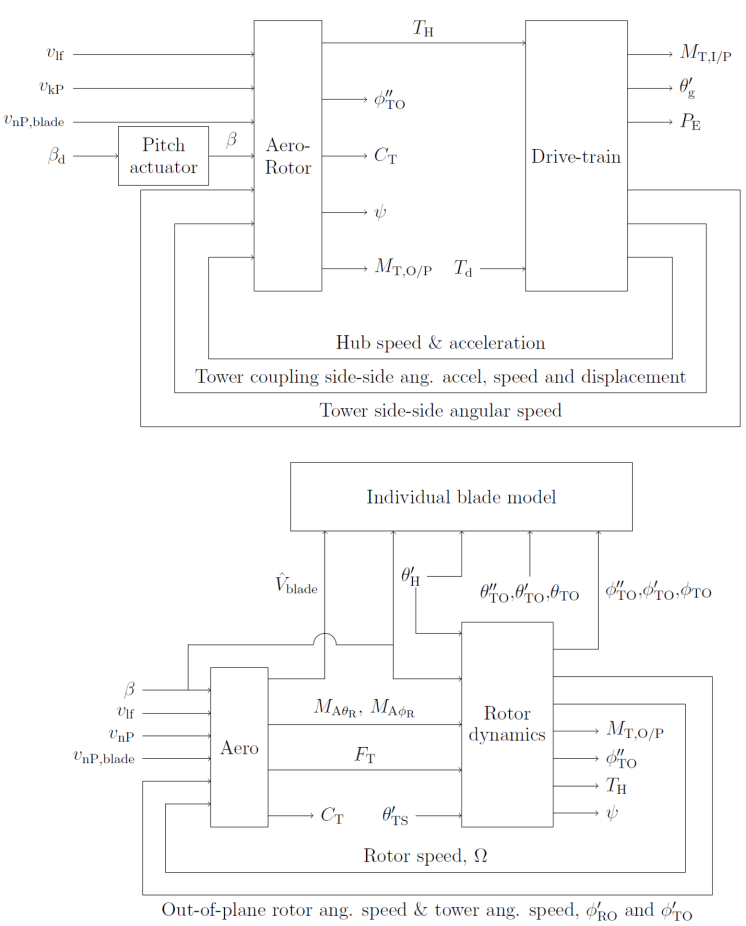

Fig. 2. Top: StrathFarm turbine diagram, with the two major components (aero-rotor and drive-train) clearly shown. Bottom: StrathFarm aero-rotor model expanded. Fig. adapted from [7]

small and so the higher frequency variation is generated independently for each turbine. Additionally, intermediate values for the wind-field low frequency component over the long sampling time intervals are constructed separately for each turbine with the correct correlation to the intervals initial and final values. For wake meandering, the lateral component of the low frequency scaler wind speed is generated (correlated again via Veers) similar to the correlated part of the turbulence in the longitudinal direction. By splitting the schotastic elements of the wind into the high and low frequency components as described a split-level integration algorithm can be used, with the low frequency (longitudinal and lateral) components numerically integrated at a 1 second step length and the high frequency components integrated at a 0.02 second step length.

\section{B. Turbine Dynamics and Turbine Controller Model}

Each wind turbine in the wind farm is modelled using a lumped parameter approach that captures the aerodynamics and the structural dynamics. The turbine model can be viewed predominantly as two dynamically linked components: the rotor and the drivetrain, as displayed on the left hand side of Fig. 2. The outputs from the wind field model in section II-A are fed into the turbine rotor model, which also receives inputs of the pitch angle, hub speed and acceleration, tower side to side displacement, angular speed, and acceleration. The aero-rotor outputs the hub torque, tower fore-aft acceleration, thrust coefficient, tower fore-aft bending moment, and the azimuthal angle. The rotor model is 
expanded at the bottom of Fig. 2 to show three components: an aerodynamics block (including dynamic inflow [15]), rotor dynamics (derived using Lagrangian methods [13] and which includes tower fore-aft dynamics), and an individual blade model [6](a lumped mass model used to calculate the edge and flap blade forces). Finally, the drive-train model has inputs of hub torque and generator torque and outputs tower side-to-side angular speed, $\theta_{T S}^{\prime}$, tower coupling sideside angular acceleration $\theta_{T O}^{\prime \prime}$, speed $\theta_{T O}^{\prime}$ and displacement $\theta_{T O}$, hub speed $\theta_{H}^{\prime}$ and acceleration $\theta_{H}^{\prime \prime}$, tower side-side root bending moment $M_{T, I / P}$, generator speed, $\theta_{g}^{\prime}$ and electrical power, $P_{E}$.

The turbine used is the SuperGen 5MW wind turbine, developed as part of the SuperGen project and broadly similar to the NREL 5MW exemplar turbine [9].

Each turbine in the wind farm has a full envelope controller (FEC) [3] that tracks the max power curve in below rated conditions from the minimum rotor speed to the maximum rotor speed. At wind speeds where the optimum rotor speed would be less than the minimum or more than the maximum speed the controller employs constant speed regions. The controller uses pitching to feather to regulate the rotor speed in above rated wind conditions, with gain scheduling based upon separability theory [10], [11], [8]. Each wind turbine is also equipped with an augmentation to the full envelope controller known as the Power Adjusting Controller (PAC) [15]. The PAC is discussed in more detail in section II-D.

\section{Wake Modelling}

When the wind interacts with a wind turbine energy is extracted from it, resulting in a reduction in the kinetic energy, and hence a reduction in the wind speed known as a wake. Whilst Computational Fluid Dynamic (CFD) approaches can be used with high fidelity, the computing time required to calculate the wakes is large and so the method is not suitable for the StrathFarm model. Instead, the popular kinematic engineering wake model of Frandsen [5] is used. The Frandsen method was previously validated against data for the Horns Rev offshore wind farm [12]. Included within the model is a wake expansion factor $\alpha$, which can simplistically be seen as setting the magnitude of the wake, with a higher value resulting in larger wake effects. A value for alpha of 0.5 is used for StrathFarm, though it is possible to change the value if desired.

StrathFarm also has the option of using a medium fidelity wake model based on the WFSim model [2], in which the model is used as a wake deficit model, applying a wake decit to the mean wind speed seen by each wind turbine at each time step. The coefficient of thrust and axial induction factor from each wind turbine model are used as the inputs to the WFSim wake model.

\section{Wind Farm Control (WFC)}

Strathfarm is designed as tool for developing wind farm controllers and so each turbine is equipped with a Power Adjusting Controller (PAC) [15] that allows the operating

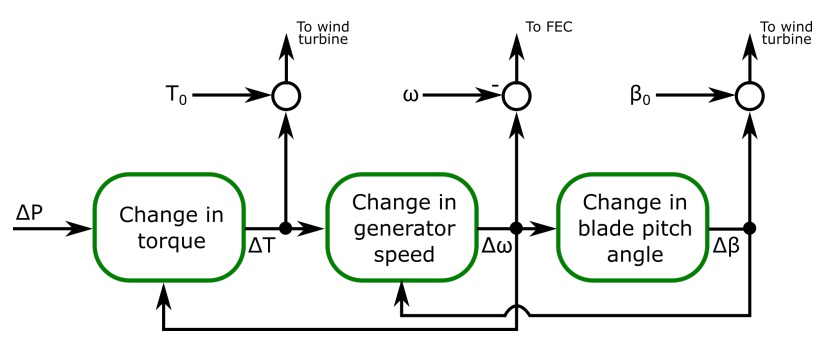

Fig. 3. A simplified flow chart of the PAC algorithm. The flow chart is a useful way to think through the operation of the PAC, with the change in power, $\Delta P$, leading to a change in torque $\Delta T$, which in turn is used to find a change in generator speed $\Delta \omega$, which drives the calculation of the change in pitch angle $\Delta \beta$.

point of the wind turbine to be altered without any changes being made to the full envelope controller, i.e. the PAC is completely independent of the full envelope controller design. Additionally, the performance of the full envelope controller is not compromised by the presence of the PAC, with minimal change to the feed-forward gain. A simplified but useful overview of the PAC's operation is shown in Fig. 3. The requested change in power $\Delta P$ is implemented through a change in the generator torque $\Delta T$, which results in a change in the generator speed $\Delta \omega$, which is in turn ameliorated (if possible) by a change in the blade pitch angle $\Delta \beta$. By effecting the change in power through the torque demand the PAC is able to deliver fast and accurate changes in power output, whilst a slower control loop minimising the error in generator speed ensures that the rotor speed is kept close to the desired value. The PAC was designed specifically to allow complete flexibility in operation, i.e. to allow any demanded change of power that is possible for the turbine to safely provide to be provided. The PAC has safety features that prevent the wind turbine's operating point from moving outside of a defined safe working envelope [15]. If the operating point moves outside of the safe working envelope then the PAC will limit its output and in some cases enter a recovery mode where the turbine is returned back to its usual operating point. When in recovery mode the PAC does not deliver the requested $\Delta P$ as changes to the torque and pitch are required to return to normal operation. The WFC is designed in a hierarchical manner as shown in Fig. 4, whereby network inputs (i.e. inputs from outside of the wind farm controller from say, the grid operator) are processed by the wind farm power controller to calculate the desired change in the wind farm's power output $\Delta P_{F a r m}$. The change in wind farm power output is input to the wind farm distributed controller, which decides how the change in power should be distributed amongst the wind turbines in the farm via the individual turbine changes in power $\Delta P_{1}, \Delta P_{2}, \ldots, \Delta P_{n}$. The turbine change in power is applied via the PACs on each turbine, which also feedback signals $S_{1}, S_{2}, \ldots, S_{n}$. The total power output of the farm is fed back to the wind farm power controller. It is important that the change in power for an individual turbine is derived from the power output of a farm of turbines (at least 4 others) in order to prevent a strong feedback loop that would negatively 


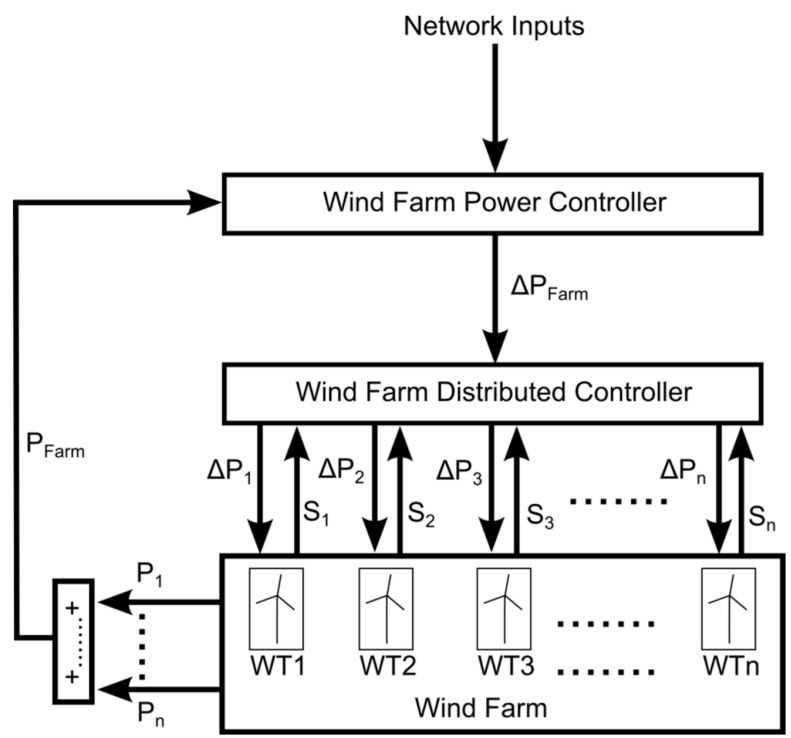

Fig. 4. Hierarchical Control Structure

impact on the operation of the wind turbine's full envelope controller. The hierarchical method used means that the WFC is able to be as flexible as possible, i.e. the WFC is minimally constrained in its functionality. By separating the wind farm power controller and the wind farm distributed controller, the two components can be designed separately and a "mix and match" approach can be used to ensure the particular goals of a particular wind farm controller are met by combining an appropriate power controller with an appropriate distributed controller.

1) Wind Farm Power Controller: In this paper a "delta control" is applied. In order to calculate the required change in power an estimate of the farm power without the WFC being applied $\left(P_{0}\right)$ is required, which is the sum of the turbine power without the PAC, calculated in the PAC by using a power curve look up table with the estimated wind speed at each turbine (including induction lag effects) as the input. Hence, the desired farm power $P_{d e s}$ is calculated by,

$$
P_{\text {des }}=K P_{0}
$$

where $K$ is a number less than 1 and greater than 0 . An error, $\epsilon$, can hence be calculated between the desired farm power and the measured farm power.

$$
\epsilon=P_{\text {des }}-P_{F} \text { arm }
$$

A PI controller is then used to minimise the error. The proportional and integral gains are found heuristically and have the values 0.8 and 0.05 respectively.

2) Wind Farm Distributed Controller: The wind farm distributed controller, referred to hereafter as simply the distributed controller, is designed to distribute the individual turbine changes in power in a manner that,

- Produces the required change in wind farm power $\Delta P_{\text {Farm }}$

- Achieves some other auxiliary goal
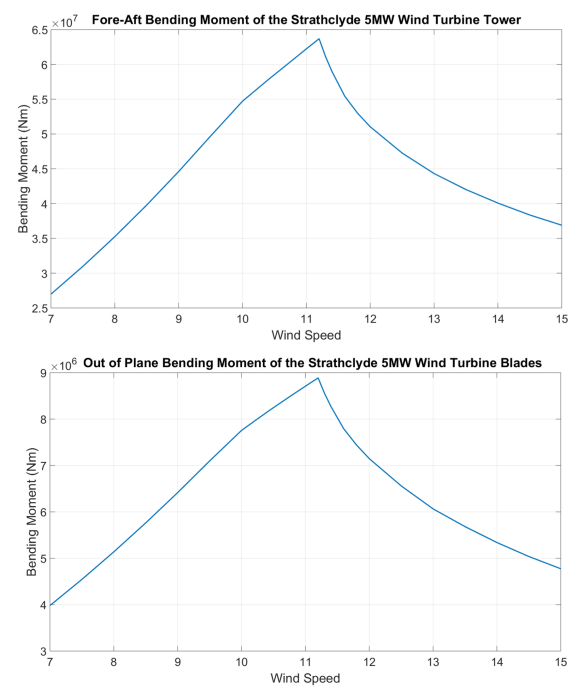

Fig. 5. Static fore-aft bending moments on the wind turbine tower (top) and static out of plane bending moments on the wind turbine blades (bottom)

For the work presented here, the auxiliary goal is to minimise the fatigue loads on the wind turbines in the farm. Fatigue loads (calculated in this paper using the approach of [14]) are dependent on the magnitude of the bending moment, the number of load cycles, and the variation in the moment over the cycle. As discussed in section II-D, the PAC effects the requested change in power through torque action, with pitch actuation used to ameliorate any induced change in rotor speed. The pitching of the blades results in a change to the fore-aft tower moment and the blade edge and flap moment. There are potentially situations whereby the blade moment is large, but the variation is small, and at these times it may therefore be beneficial to not curtail a wind turbine experiencing high bending moments and at times it may even be benecial, at least hypothetically, to request more power from a turbine in order to maintain the bending moments or at least to minimise any changes to them. Whilst the loads on a wind turbine's blades and tower are dynamically changing and therefore, a methodology that takes into account the measured loads when distributing the turbine changes in power has some merit, the loads on the blades and tower are not typically measured on a wind turbine. Hence, a simpler methodology, based on the static loads on the turbine is applied here. The static out of plane loads on the wind turbine blades and fore-aft loads on the tower are shown in Fig. 5. It can be seen that both out-of-plane blade bending moments and tower fore-aft bending moments increase from a minimum at low wind speeds towards a peak at the rated wind speed (just over $11 \mathrm{~m} / \mathrm{s}$ ), before reducing again as the wind speed continues to increase. The reduced moments in low wind conditions are due to lower thrust from the wind, whilst the reduced moments in higher wind speeds are due to the wind turbine blades pitching to feather and hence reducing the thrust coefficient of the rotor. It is clear from Fig. 5 that pitching the blades can have a significant 

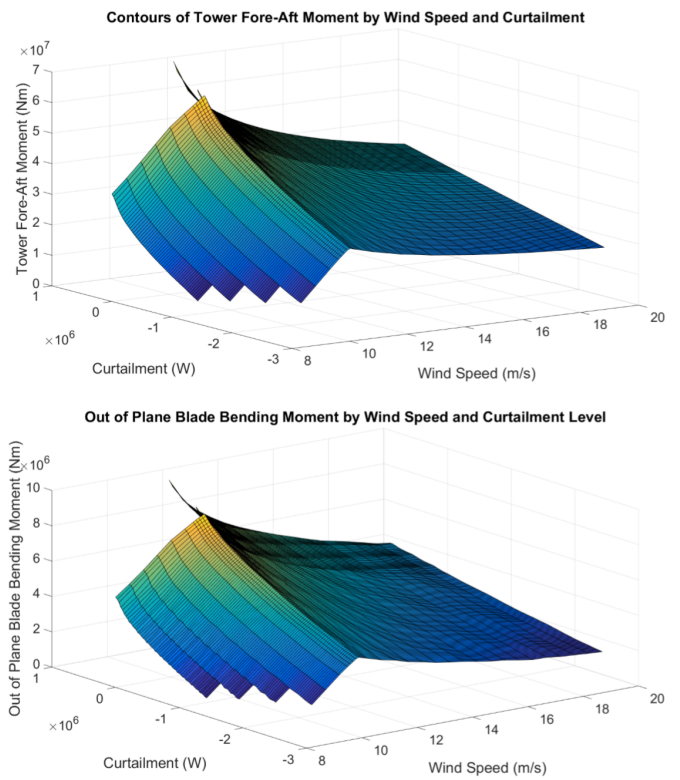

Fig. 6. Surface of fore-aft tower (top) and out-of-plane blade (bottom) static bending moments for a range of wind speeds and curtailment levels

effect on the structural bending moments and it is true that pitching the blades due to a power reduction via the PAC results in a similar change in thrust force and hence could potentially reduce the blade out-of-plane and tower fore-aft loads. Investigating the hypothesis that curtailing through the use of the PAC on each turbine can influence the structural loads, contour plots of the relevant moments for a range of static curtailments and wind speeds are produced as shown in Fig. 6. Given the information shown in Fig. 5 and Fig. 6 it is hypothesised that allocating the change in power in a manner that evens the loads over the turbines in the wind farm could lead to an overall reduction in the fatigue loads. The strategy designed to achieve this aim is for wind turbines in the farm to follow the contour plot shown in Fig. 7 as a baseline strategy where the baseline change in power $\Delta P_{n B a s e}$ is dependent upon the wind speed as estimated by the turbine's PAC. Note that because the wind speed estimate is highly decoupled from the turbine dynamics there is no strong feedback loop induced around the turbine. Clearly following the baseline on its own will not necessarily result in the correct change in power and so the required additional change in power $\Delta P_{n A d d}$, which may be positive or negative, is included for each turbine such that, for the $n$th wind turbine in a farm of $N$ available turbines,

$$
\Delta P_{n}=\Delta P_{n \text { Base }}+\Delta P_{n A d d}
$$

The required additional change in power $\Delta P_{a d d}$ is allocated to the wind turbines proportionally to the estimated wind speed from the PAC squared $\left(\hat{V}^{2}\right)$. Hence the total change in power for the $n$th wind turbine in a farm of $N$ available

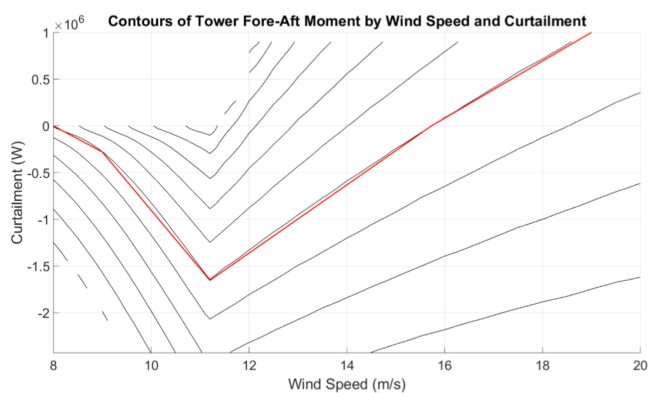

Fig. 7. A plot of contours of constant tower fore-aft moment by curtailment and wind speed with the proposed baseline curtailment strategy

turbines,

$$
\Delta P_{n}=\Delta P_{n \text { Base }}+\frac{\Delta P_{\text {Farm }}-\sum_{n=1}^{N} \Delta P_{n \text { Base }}}{\sum_{n=1}^{N} \hat{V}_{n}^{2}}
$$

For comparison, a second strategy is also simulated, whereby the change in the farm power is simply evenly spread amongst the available turbines in the farm. This first methodology is referred to herein as the Intelligent Distributed Controller or (IDC), whilst the second is the Evenly Distributed Controller (EDC).

Because the controller contains integral action, it is necessary to include anti-wind up to prevent integral wind up when the limits of the actuator (in this case the PAC and turbine) are exceeded. The back calculation method described in [15] is used for this purpose. The rate of change of power is also limited to prevent unwanted loads on the turbine from sudden changes in torque. Note that only "available" turbines are considered for the distributed controller. An available turbine is one that,

- Has an effective wind speed estimate above the minimum wind speed (in this case $6 \mathrm{~m} / \mathrm{s}$ )

- Is in a safe part of the operational envelope as determined by the signals from the PAC [15]

- Is not currently undergoing the recovery process to return to normal operation

\section{WIND FARM LAYOUT AND ENVIRONMENTAL CONDITIONS}

The simulation results presented in this paper use a wind farm of 16 SuperGen 5MW wind turbines arranged in a $4 \times 4$ square. Whilst simple, such an arrangement is common for offshore wind turbines. Simulations are conducted for all permutations of the following conditions:

- Mean wind speeds $9 \mathrm{~m} / \mathrm{s}, 12 \mathrm{~m} / \mathrm{s}, 15 \mathrm{~m} / \mathrm{s}$, and $18 \mathrm{~m} / \mathrm{s}$

- Wind directions $0^{\circ}$ (perpendicular to the side of the square), $22.5^{\circ}$, and $45^{\circ}$ (note that due to the symmetric arrangement of the wind farm these directions are also valid for their value plus $90^{\circ}$, plus $180^{\circ}$, and plus $270^{\circ}$ ).

- Turbulence levels of $10 \%$

- Power reduction of $10 \%$ of normal farm power output at the wind speeds experienced by the farm. 
- Two WFC designs (IDC and EDC as described in section II-D) and the base case where no WFC is used.

\section{Results}

For each simulation conducted the two specific aims of the controller as stated in section II-D.2 must be considered, specifically,

- Was the delta control accurate?

- What was the effect on the tower fore-aft and blade out-of-plane fatigue loads

To assess the tower fore-aft and blade out-of-plane fatigue loads the damage equivalent loads (DELs) for each case are calculated. The fatigue loads for each wind speed and direction combination are shown in Table I. The DELs are shown as the percentage difference from the non-WFC case. All the percentage changes are negative, so in all cases the DELs were decreased through the control action. For wind speeds of $9 \mathrm{~m} / \mathrm{s}, 15 \mathrm{~m} / \mathrm{s}$ and $18 \mathrm{~m} / \mathrm{s}$ the IDC controller out performs the EDC controller in both tower and blade DEL reduction by as much as $13.3 \%$ and $16.3 \%$ respectively. At $12 \mathrm{~m} / \mathrm{s}$ the EDC outperforms the IDC. It is not clear at this time why this is the case and whilst performance is still good for IDC, future work should be undertaken to identify the reason for the difference.

To assess the accuracy of the delta control the power output of the controlled cases are compared to $90 \%$ of the power of the non-controlled case (perfect delta control) and the mean error is calculated. The results are shown at the bottom of Table I. The mean percentage error in the change in power is high for low wind speeds, whilst at high wind speeds the error is much smaller. The reason for the large error at low wind speeds is that the change in farm power $\Delta P_{\text {Farm }}$ does not take into account the changes in the wakes of the wind turbines caused by altered operation. The error is largest in the case of the $9 \mathrm{~m} / \mathrm{s}$ average wind speed simulations, as in below rated wind conditions a reduction in the effective wind speed has a cubic relationship to the reduction in power. In simulations well above rated the wakes have a minimal effect on power as the turbine is already limiting it's power to the rated value through pitch actuation and hence the mean error is much smaller. The effect is highlighted in Fig. 8, which shows plots from the simulation at $9 \mathrm{~m} / \mathrm{s}$ and 0 degrees wind. The upper plot in Fig. 8 shows the farm power output without wind farm control, the "perfect" total farm output of $90 \%$ of farm power output without wind farm control and the power output with IDC and EDC. It is clear that both IDC and EDC have substantial errors. In the middle plot the estimate of power without WFC is shown to be accurate when no WFC is in use. When using IDC or EDC however, the estimate is no longer accurate. The wind speed estimate of the PAC is highly decoupled from the turbine and has been shown to be accurate when the PAC is in operation [15], however the average wind speed estimate across the farm is shown to be increased in the lower plot of Fig. 8. The clear explanation is that the reduction in power of upstream wind turbines has increased the wind speed in downstream turbines, leading to a change in the wind speed
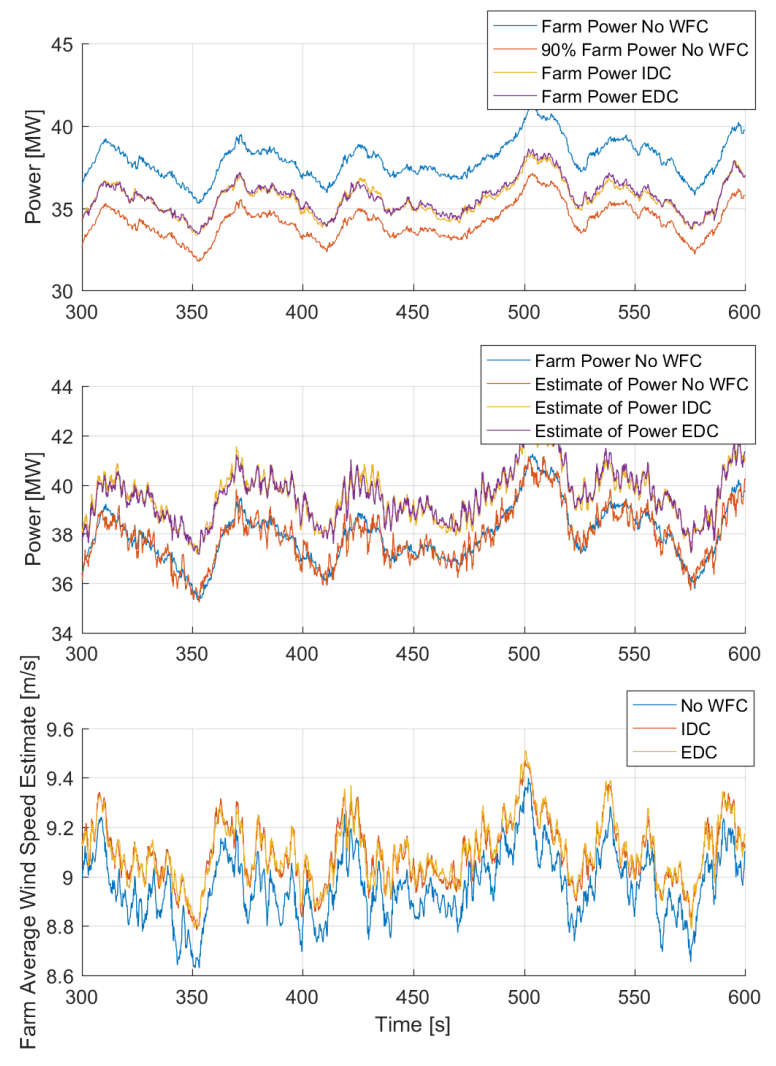

Fig. 8. Measured Farm Power, Estimated Farm Power and Estimated Wind Speed with Different Controllers

experienced by the downwind turbines and hence an increase in the wind speed estimate, leading to an over-estimate of the power output without WFC in use.

\section{CONCLUSIONS}

A wind farm controller designed to minimise the structural loads on the wind turbines in the farm whilst providing a "delta" change in power of $10 \%$ of the non controlled output was designed and tested using the StrathFarm wind farm simulation package. At higher wind speeds the delta power tracking was accurate, however, at lower wind speeds the tracking is less accurate, with a mean error of $\mathrm{X} \%$ across all simulated directions at $9 \mathrm{~m} / \mathrm{s}$ mean wind speed. The poorer performance in low wind speeds is due to the diminished wakes of the upstream turbines increasing the power output of the down stream turbines. Future work should focus on how to remedy this error, perhaps by basing the change in power on the estimated power of the upwind turbines only.

The IDC strategy, which pre-allocated an initial change in power on each turbine based on static thrust modelling and then allocated the remaining change in power proportional to the square of wind speed showed excellent reductions in tower and blade DELs. Reductions of between 5\% and $23 \%$ compared to no power reduction were observed. The reduction in DELs was greater than the more simplistic 


\begin{tabular}{|c|c|c|c|c|c|c|c|c|c|c|c|c|}
\hline Mean Wind Speed $[\mathrm{m} / \mathrm{s}]$ & \multicolumn{3}{|c|}{9} & \multicolumn{3}{|c|}{12} & \multicolumn{3}{|c|}{15} & \multicolumn{3}{|c|}{18} \\
\hline Direction [deg] & 0 & 22.5 & 45 & 0 & 22.5 & 45 & 0 & 22.5 & 45 & 0 & 22.5 & 45 \\
\hline IDC Change in Tower DELs [\%] & -7.9 & -18.8 & -14.5 & -8.3 & -14.9 & -13.3 & -22.4 & -23.1 & -22.2 & -7.0 & -7.6 & -5.5 \\
\hline EDC Change in Tower DELs [\%] & -1.5 & -10.7 & -7.7 & -14.0 & -16.8 & -16.4 & -10.1 & -9.8 & -10.3 & -4.3 & -4.4 & -2.2 \\
\hline IDC Change in Blade DELs [\%] & -14.4 & -20.4 & -19.1 & -1.7 & -9.0 & -8.4 & -23.4 & -23.0 & -23.0 & -6.8 & -7.2 & -7.0 \\
\hline EDC Change in Blade DELs [\%] & -6.1 & -3.7 & -6.2 & -9.0 & -11.1 & -12.8 & -7.1 & -6.8 & -7.3 & -3.5 & -4.0 & -3.6 \\
\hline IDC Mean Error [\%] & 37.1 & 39.8 & 42.2 & 22.4 & 7.3 & 13.8 & -3.2 & -1.8 & -0.2 & 5.0 & 4.7 & 5.4 \\
\hline EDC Mean Error [\%] & 40.1 & 43.4 & 45.3 & 23.3 & 7.4 & 13.8 & -0.52 & -0.54 & -0.54 & -1.4 & -1.3 & -1.35 \\
\hline
\end{tabular}

TABLE I

DAMAGE EQUIVALENT LOADS FOR EACH STRATEGY COMPARED TO NO REDUCTION IN POWER AND MEAN ERROR FOR EACH STRATEGY

EDC strategy that simply divided the required change in power equally between available turbines for all wind speeds except $12 \mathrm{~m} / \mathrm{s}$, at which mean wind speed the reductions were slightly reduced compared to EDC.

Whilst the approach here requires refinement to account for the wake effects that cause errors in the change in power, which would be an interesting area for further work, the principle of distributing the changes in turbine power based on the thrust curve and the wind speed is shown to be very promising for reducing loads.

\section{REFERENCES}

[1] G.B. National Grid Status - https://www.gridwatch.templar.co.uk/.

[2] Sjoerd Boersma, Bart Doekemeijer, Mehdi Vali, Johan Meyers, and Jan-Willem van Wingerden. A control-oriented dynamic wind farm model: Wfsim. Wind Energy Science, 3(1):75-95, 2018.

[3] Aristeidis Panagiotis Chatzopoulos. Full Envelope Wind Turbine Controller Design for Power Regulation and Tower Load Reduction. $\mathrm{PhD}$ thesis, University of Strathclyde, 2011.

[4] Paul A. Fleming, Jacob Aho, Andrew Buckspan, Erik Ela, Yingchen Zhang, Vahan Gevorgian, Andrew Scholbrock, Lucy Pao, and Rick Damiani. Effects of power reserve control on wind turbine structural loading. Wind Energy, 19(3):453-469, mar 2016.

[5] Sten Frandsen, Rebecca Barthelmie, Sara Pryor, Ole Rathmann, Søren Larsen, Jørgen Højstrup, and Morten Thøgersen. Analytical modelling of wind speed deficit in large offshore wind farms. Wind Energy: An International Journal for Progress and Applications in Wind Power Conversion Technology, 9(1-2):39-53, 2006.

[6] Maria Lourdes Gala-Santos. Aerodynamic and wind field models for wind turbine control. Phd thesis, University of Strathclyde, 2019.

[7] Alex Giles, Daan van der Hoek, Gabriele Bedon, Karl Merz, Martin Kühn, Mehdi Vali, Olimpo Anaya-Lara, Stoyan Kanev, Vlaho Petrovíc, Davide Trabucci, and William Leithead. Offshore array control, irp wind work package 6.3 - deliverable number 63.4. Technical report, Integrated Research Programme on Wind Energy - lead partner University of Strathclyde, 2018.

[8] Peter Jamieson, WE Leithead, and Maria Gala-Santos. The Aerodynamic Basis of a Torque Separability Property. Proc. European Wind Energy Association Conference 2011, 2011.

[9] Jason Jonkman, Sandy Butterfield, Walter Musial, and George Scott. Definition of a 5-mw reference wind turbine for offshore system development. Technical report, National Renewable Energy Lab.(NREL), Golden, CO (United States), 2009.

[10] W E Leithead and D J Leith. On the Separability of Wind Turbine Rotor Aerodynamics. Technical report, University of Strathclyde, 2000.

[11] WE Leithead, DJ Leith, F Hardan, and H Markou. Global gainscheduling control for variable speed wind turbines. In EWEC conference, pages 853-856, 1999.

[12] Martin Méchali, Rebecca Barthelmie, Sten Frandsen, Leo Jensen, and Pierre-Elouan Réthoré. Wake effects at horns rev and their influence on energy production. In European wind energy conference and exhibition, volume 1, pages 10-20. Citeseer, 2006.

[13] Victoria Neilson. Individual Blade Control for Fatigue Load Reduction of Large-scaled Wind Turbines: Theory and Modelling. Mphil, University of Strathclyde, 2010.
[14] Charles Plumley. The Smart Rotor Wind Turbine. Phd thesis, University of Strathclyde, 2015.

[15] Adam Stock. Augmented control for flexible operation of wind turbines. Phd thesis, University of Strathclyde, 2015.

[16] Paul S Veers. Three-dimensional wind simulation. Technical report, Sandia National Labs., Albuquerque, NM (USA), 1988. 